\title{
O PROCESSO ENSINO-APRENDIZAGEM NA VERIFICAÇÃO DA PRESSÃO ARTERIAL
}

\author{
RELATED TEACHING-LEARNING PROCESS TO THE TECHNIQUE \\ OF VERIFICATION OF THE ARTERIAL PRESSURE \\ EL PROCESO ENSEÑANZA - APRENDIZAJE EN LA VERIFICACIÓN DE \\ LAPRESIÓNARTERIAL
}

Thereza Maria Magalhães Moreira1
Taciana Cavalcante de Oliveira ${ }^{2}$
Thelma Leite de Araújo ${ }^{3}$

RESUMO: A enfermeira é considerada a profissional que obtém dados mais fidedignos nas medidas indiretas de pressão arterial (PA) por induzir menos reaçōes de alerta no cliente, geradoras de valores falsamente elevados. Porém, muitas vezes, ela apresenta lacunas no conhecimento do assunto, tanto nos aspectos técnicos, como nos anátomo-fisiológicos. Isso prioriza o desenvolvimento de estratégias de ensino, visando minimizar falhas e tornar o processo de aprendizagem efetivo. Objetivando promover o conhecimento da técnica de aferição da PA junto a um grupo de pesquisa, realizou-se uma reciclagem e aplicou-se questionário avaliativo do conhecimento do grupo antes e após a mesma. Evidenciou-se a eficácia desse método, pois $100 \%$ da amostra (nove) referiu contribuição para aprimoramento da fundamentação teórico-prática, diminuindo dificuldades oriundas da verificação da PA. Conclui-se que, sendo as açōes de educação em saúde extremamente válidas e necessárias, o enfermeiro, como educador que é, necessita reciclar seu conhecimento e aprimorar suas técnicas continuamente.

PALAVRAS-CHAVE: ensino-aprendizagem, pressäo arterial, Enfermagem.

\section{INTRODUÇÃO}

Um dos grandes problemas de saúde pública no Brasil, nos dias atuais, é a hipertensão arterial (HA), tanto pela sua prevalência no pais, como pela detecção quase sempre tardia da doença. Seu controle constitui um problema complexo devido à magnitude e seriedade do assunto e por representar um grande desafio ao nosso sistema de saúde, ainda muito alicerçado no modelo curativo e farmacêutico.

A hipertensão arterial é uma condição clínica de natureza multifatorial, caracterizada por niveis de pressão arterial sistólica e/ou diastólica elevada. Seus niveis variam entre as pessoas e oscilam durante o dia devido a fatores posturais, sensoriais, metabólicos, musculares, térmicos e etários (HAS, 1996, Porto; Rassi, 1990).

A pressão arterial (PA) é elemento fundamental na dinâmica da circulação sangüinea, pois mantém o aporte de oxigênio necessário à nutrição dos tecidos, dos órgãos e da própria vida humana. Mas, em excesso, a PA pode provocar enormes danos à saúde $\mathrm{e}$, inclusive, a

\footnotetext{
${ }^{1}$ Mestranda em Enfermagem pela Universidade Federal do Ceará, bolsista da CAPES.

2 Doutora em Enfermagem, Adjunto do Departamento de Enfermagem da Universidade Federal do Ceará e Coordenadora do Projeto "Cuidando de Pessoas com Alterações da Pressäo Arterial".

${ }^{3}$ Especializanda em Enfermagem, bolsista de aperfeiçoamento do CNPq.
} 
morte. Medidas para seu controle têm importante papel na detecção precoce, necessária à prevenção de sua morbi-mortalidade e das complicações, diminuindo custos com tratamentos crônicos e melhorando a qualidade de vida dos acometidos.

A primeira detecção da existência de pressão arterial data de 1733 e foi realizada pelo reverendo inglês Stephen Hales, que canulou a carótida de uma jumenta e observou o sangue subir até $290 \mathrm{~cm}$ de altura. Mas foi em dezembro de 1896 (123 anos depois) que Scipione Riva Rocci apresentou ao mundo o que chamou de "nuovo sphygmomano-metro", semelhante aos instrumentos de verificação da PA atuais. Portanto, faz mais de 100 anos a invenção que revolucionou as ciências da saúde. A criaçăo de Rocci foi aperfeiçoada em 1905 por Nicolai Korotkoff, que introduziu o sistema auscultatório para as medidas de pressão sistólica e diastólica. Em 1939, surgiu o conceito de hipertensão arterial como fator de risco independente para as doenças cardiovasculares (DCV). Essa concepção é fruto da introdução da verificação da pressão arterial como rotina em uma seguradora em 1917. No entanto, foi somente no final da década de 40 e início dos anos 50 , que os primeiros estudos epidemiológicos sobre hipertensão arterial começaram a ser realizados pelos Ministérios da Saúde da Europa e Estados Unidos da América (EUA) com o objetivo de organizar programas de saúde pública, sendo as campanhas de conscientização iniciadas na década de 60 (Lolio 1990; Araújo; Arcuri; Martins, 1998).

Até hoje são realizados estudos visando compreender a melhor maneira de verificação da PA. Zanella et al. (1992) afirmam que a classificação da hipertensão quanto à gravidade, utilizando-se referenciais numéricos, é útil porque, em geral, as complicaçōes ocorrem na proporção direta dos niveis tensionais. No entanto, a ausência ou presença de lesão nos órgãosalvo (coraçăo, rins, cérebro, artérias e retina) é ainda muito importante na avaliação clinica da severidade do processo hipertensivo. Evidentemente, um paciente que apresente proteinúria e hipertrofia cardiaca apresenta uma forma severa de hipertensão arterial, mesmo que os niveis tensionais năo estejam tão elevados. Por outro lado, um paciente com niveis muito elevados de pressão arterial deve também ser considerado como portador de forma severa da hipertensăo e receber tratamento intensivo, pela grande posssibilidade de lesão nos órgãos-alvo. Normalmente, nos quadros de hipertensão arterial, ambas as pressões, sistólica e diastólica, estão elevadas, porém o achado de hipertensão sistólica isolada não é incomum e deve ser tratado.

A importância da enfermeira na aferição da PA vem sendo reconhecida há mais de 50 anos. Nos últimos anos esse papel se ampliou muito além do simples procedimento de verificação da pressão, pois diversos estudos têm demonstrado que tanto no desenvolvi-mento de protocolos de pesquisa, como na prática de atendimento à saúde, os niveis de $\mathrm{PA}$, aferidos pelas enfermeiras sofrem menos variações que os obtidos por médicos.

Araújo (1994) em pesquisa desenvolvida com enfermeiros da área de cardiologia, identificou lacunas importantes no conhecimento dessa categoria sobre a medida indireta da PA. Cita, inclusive, que enfermeiras que trabalham com clientes portadores de alterações cardiovasculares, nos quais o controle fidedigno da PA assume um aspecto importante, desconhecem aspectos relativos à técnica, ao instrumental e aos aspectos anátomo-fisiológicos envolvidos. Questiona, ainda, a responsabilidade das escolas e dos programas de educação continuada, sugerindo a necessidade do desenvolvimento de novas formas de ensino-aprendizagem. A atualização dos conhecimentos inclui, em especial, o acompanhamento dos estudos relativos às recomendaçōes sobre a medida da pressão arterial. A discussão dos aspectos polêmicos envolvidos na técnica de mensuraçăo não deve ser restrita ao meio acadêmico, mas envolver profissionais que atuam junto à clientela que apresenta alterações da PA.

O processo ensino-aprendizagem mostra-se complexo por sofrer influência de fatores externos, dentre eles: metodologia de ensino, relação aluno- professor e a própria estrutura 
fisica das instituiçōes de ensino. Sabemos das dificuldades sobre-humanas que as universidades públicas, em especial, vêm enfrentando com a desvalorizaçăo do ensino, os baixos salários dos professores e as parcas condições de trabalho em que estes desempenham suas atividades cotidianas.

Para Mizukami (1986) o ensino e a aprendizagem têm significado amplo, năo havendo restriçōes às suas situaçōes formais, desta forma acredita-se que o ensino não deve ser realizado apenas nas salas de aula.

No tocante à técnica de verificaçăo da PA, essa realidade não é diferente. Há a necessidade fremente de estimular o desenvolvimento profissional capacitado para a deteç̧ão precoce e acompanhamento da hipertensão.

Diante de todo o exposto no inicio deste trabalho, entende-se ser importante a realização de estudos que visem a detecção precoce da doença, seu acompanhamento e evolução. Sentiuse a necessidade de treinar um modelo de ensino do procedimento da medida da pressão arterial a ser desenvolvido com acadêmicos de enfermagem e enfermeiros. Resolveu-se, então, testar um modelo ou uma estratégia de ensino com a finalidade de aprofundar e promover o conhecimento da técnica de aferição da pressão arterial junto a um grupo de pesquisa.

\section{OBJETIVOS}

GERAL:

- Promover o conhecimento da técnica de aferição da pressão arterial junto a um grupo de pesquisa.

\section{ESPECIFICOS:}

- Aprimorar a técnica de verificação da PA em um grupo de pesquisa;

- Avaliar o conhecimento apreendido sobre verificação da pressão arterial pela clientela em estudo em dois momentos distintos: pré e pós-treinamento, comparando o conhecimento obtido nos dois momentos.

\section{REFERENCIAL TEÓRICO}

\section{O PROCESSO ENSINO-APRENDIZAGEM}

A aprendizagem é um processo integrado no qual o eu holistico é mobilizado de forma orgânica qualitativa, e pelo qual a pessoa fica melhor preparada para novas aprendizagens, não havendo, portanto, um aumento quantitativo de conhecimentos, mas uma transformação estrutural da inteligência da pessoa. $O$ aluno é o agente essencial desse processo. Já o processo de ensino é pragmático, ou seja, é um mecanismo pelo qual se tenta alcançar determinados objetivos através de estratégias seqüenciais. Dessa maneira, o processo de ensino consiste em planejar, orientar e acompanhar a aprendizagem do aluno (Bordenave; Pereira, 1977).

Os alunos são diferentes entre si e necessitam de diferentes tipos de aprendizagem para seu próprio desenvolvimento. Para que se obtenha uma aprendizagem eficaz, é indispensável que se considere as capacidades de cada um ao selecionar e organizar os procedimentos de ensino, o que é uma tarefa dificil e complexa (Turra et al., 1975). Mas Novaes (1992) afirma que os processos criativos poderiam ser implantados em todas as disciplinas, proporcionando ao aluno uma abordagem inovadora do conteúdo.

A aprendizagem é uma mudança na disposição ou capacidade do homem, modificação essa que pode ser inferida comparando-se o comportamento possivel antes e após o individuo ser colocado em uma situação de aprendizagem. Dessa maneira, o processo de aprendizagem se realiza quando a situação estimuladora afeta de tal maneira o aprendiz que seu desempenho 
é modificado após o contato com essa situação. É importante considerar as habilidades já adquiridas antes mesmo do processo de aprendizagem (Gagné, 1973).

As diversas crises econômicas, mudanças nas politicas de saúde, novas necessidades da demanda, desenvolvimento cientifico-tecnológico, novos instrumentos, além de outros, foram integrando o cotidiano da enfermagem e imprimindo alterações em sua prática assistencial e no ensino. Para que os docentes e a equipe de enfermagem que atua na assistência possam elaborar e trocar conhecimentos entre si visando atingir um cuidado qualitativamente mais apropriado, têm que se atentar à dinâmica das relações que văo se estabelecendo no cotidiano profissional, às tradiçöes e novas tecnologias, materiais e intelectuais que instrumentalizam o processo de trabalho e às necessidades apresentadas nesse ambiente. E diz que é mister saber desenvolver as técnicas visando a superaçăo do efeito tecnicista da própria atividade, numa internalização da mesma, uma vez que as técnicas são um conteúdo fundamental na formação da enfermeira, pois compõem um instrumento de seu trabalho (Collet; Rocha, 1996).

\section{PADRONIZAÇÃO NA MEDIDA DA PRESSÃO ARTERIAL}

Sempre houve uma preocupaçăo dos estudiosos referente à padronização na medida da PA. Araújo (1994) cita que a necessidade de tornar o método não invasivo auscultatório mais preciso incentivou a formação de especialistas, designados pela Associação Americana de Cardiologia para a construção de padrōes utilizados na mensuração da PA. A primeira publicação contou com a participação de vários estudiosos renomados e abordou temáticas relativas à padronização do instrumental, posição do cliente e método de adaptação do manguito. Este trabalho ficou conhecido como padronização da medida da pressão arterial, foi publicado em 1939 e avaliado nos Estados Unidos e Europa em décadas seguintes, considerando o conhecimento ao longo dos anos, relativo às possiveis fontes de erro na aferição da PA, referente ao observador, ao cliente e ao instrumental. A padronização tem incorporado este conhecimento e as revisões têm divulgado as novas determinaçőes.

A seguir encontra-se um quadro expositivo da técnica de verificação da PA recomendada pela última revisão publicada pela American Heart Association (Perloff, 1993). 
VERIFICANDO A PA

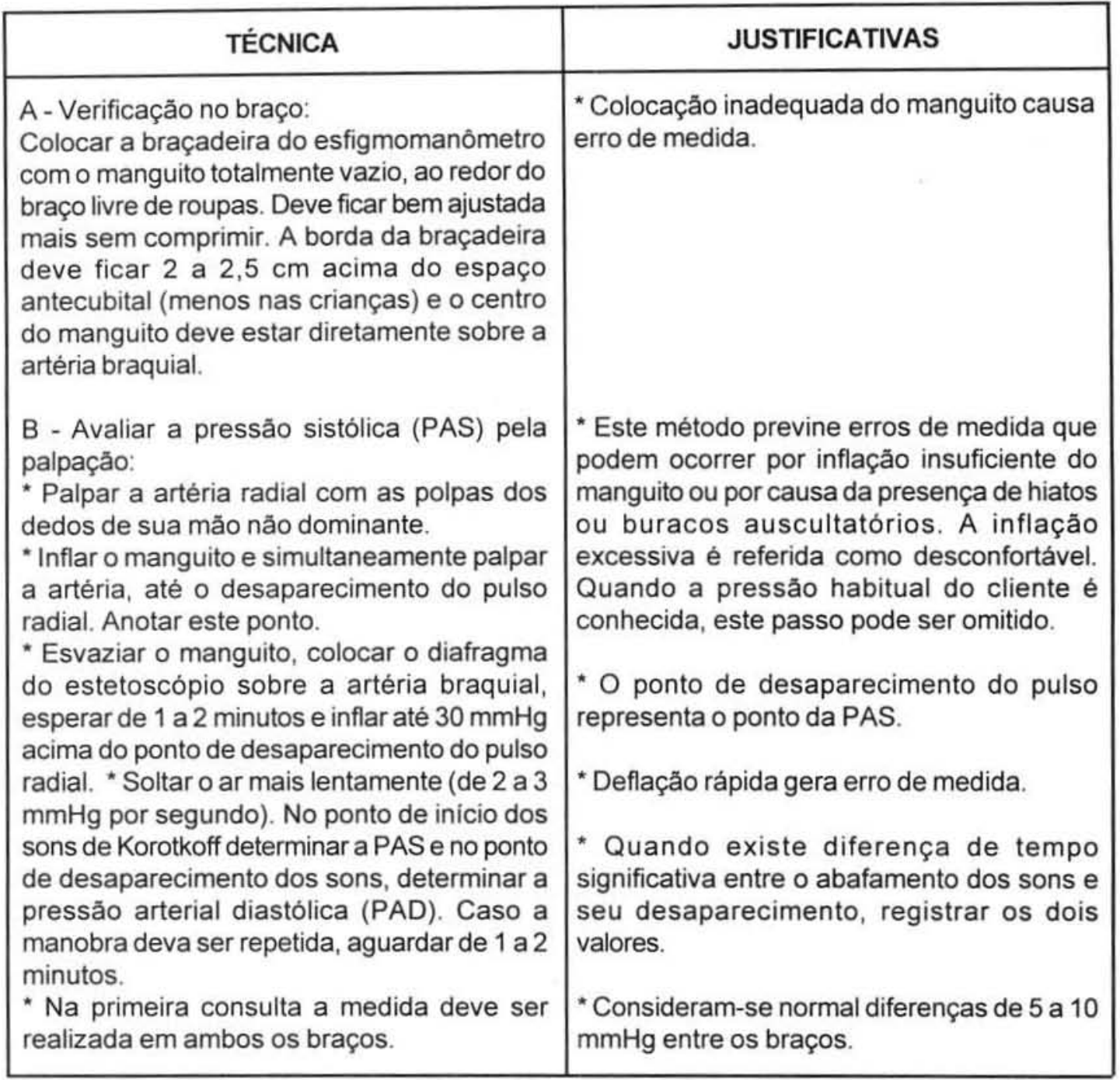

\section{ENFERMAGEM NA ASSISTÊNCIA AOS HIPERTENSOS}

A enfermagem no controle da HA vem prestando cuidados aos individuos com hipertensão com o intuito de controlar a PA e evitar possiveis complicações. Além disso, para indivíduos que apresentam fatores de risco e déficit de conhecimento que contribuem para o desencadeamento da hipertensăo, a enfermeira mantém, através do sistema apoio-educação, esclarecimentos, possibilitando a promoção e recuperação da saúde do cliente.

O déficit de conhecimento da técnica de aferição da PA prejudica no tratamento dos hipertensos, pois diante de técnicas inadequadas poderemos ter valores incorretos, dificultando a avaliação fidedigna da situação clínica do individuo.

ENFERMAGEM E A TÉCNICA DE VERIFICAÇÄO DA PA (esfigmomanometria)

A investigação no campo da esfigmomanometria vem mostrando que a enfermeira é o 
elemento da equipe de saúde mais indicado para executar a medida da PA. O fato da enfermeira causar menor reação de alerta no cliente é fundamentado nos estudos realizados por Mancia et al. (1987), que compararam, em pacientes internados, o aumento da PA provocado pela entrada do médico na enfermaria com o provocado pela enfermeira. A técnica indireta de aferição da PA pode se tornar mais imprecisa devido à reação de alerta. A pseudo hipertensão durante medida casual em consultórios, clinicas e enfermarias em alguns clientes ou pacientes é fenômeno conhecido e demonstrado.

As enfermeiras vêm constantemente se firmando como responsáveis pela detecção precoce da $\mathrm{HA}$ desconhecida e não controlada e pelo controle do quadro hipertensivo já conhecido, sendo particularmente atuantes na educação dos pacientes, reforçando a importância do controle continuo e a longo prazo da PA (Hill; Mccombs, 1981).

Assim, a enfermeira tem tido um papel preponderante nos programas de hipertensão arterial, estando perfeitamente apta a tratar com pacientes hipertensos pela sua formação acadêmica, que requer o trabalho com grupos, pela a observância de princípios como interdisciplinaridade e multiprofissionalismo, além da regulamentação pelo Conselho Federal de Enfermagem (COFEN) da consulta e prescriçăo de enfermagem no cuidado a pacientes hipertensos em acompanhamento (Conselho Regional de Enfermagem/COREN-CE, 1994: Lei $7.498 / 86$, regulamentada pelo Decreto $N .{ }^{\circ} 94.406 / 87$ ). Acredita-se que muito importam todas as ações desenvolvidas pela enfermagem no âmbito da hipertensão, assim como seus registros e teorizações.

A necessidade da enfermeira detectar dados hemodinâmicos precisos e de interpretálos com segurança é indiscutivel, o que requer sólido conhecimento teórico e prático no campo da esfigmomanometria a fim de garantir a tomada de decisão e encaminhamentos corretos. No entanto, a medida da PA por ser considerada um procedimento corriqueiro há anos, não é tema dos programas de educação continuada. Sabe-se que até recentemente, a despeito do desenvolvimento tecnológico e das pesquisas no assunto, pouco foi acrescentado ao conhecimento adquirido nos cursos de graduação. O pobre conhecimento no campo da esfigmomanometria já foi referido por Arcuri (1989), ao identificar um desconhecimento preocupante por parte dos profissionais da área de saúde. Isso foi mais recentemente confirmada por Araujo (1994), que constatou importante lacuna no conhecimento da medida indireta da pressão arterial, entre enfermeiros atuantes na área de cardiologia.

Considerando a hipertensão como um dos grandes fatores de risco para doenças cardiovasculares, um dos importantes agravantes no perfil de morbi-mortalidade em todas as regiōes do mundo e que a medida precisa da PA é um dos parâmetros essenciais a sua detecção precoce, considera-se fundamental a atualização da enfermagem neste âmbito.

\section{METODOLOGIA}

O estudo foi desenvolvido no Departamento de Enfermagem da Universidade Federal do Ceará. Propõe-se uma abordagem exploratório-descritiva.

A população constou dos quinze integrantes de um projeto de pesquisa, que tem, entre outros, o objetivo de:

- Identificar casos novos de hipertensão, relacionando os niveis de PA com a presença de fatores de risco e variáveis constitucionais, sociais, étnicas e econômicas.

- Desenvolver açōes educativas-assistenciais para clientes com diagnóstico de HA e para os que pertencem a grupos de risco, contribuindo para a adesão ao tratamento proposto.

A amostra foi representada por nove integrantes do Projeto cuidando de pessoas com 
alterações da pressão arterial presentes à reunião do dia 22 de abril de 1998.

O processo ensino-aprendizagem foi realizado através de aula expositiva, discussão em grupo, bem como aplicação prática da técnica atual de mensuração da PA.

A fonte de coleta de dados foi do tipo primária, sendo o levantamento de dados ocasional. O processo de coleta foi do tipo interrogatório, na forma de questionário tipo cédula, aplicado antes e após o desenvolvimento de um processo de reciclagem explicativa, que abordou o método atual de aferição da pressão arterial (PA). O instrumento utilizado foi do tipo semiestruturado.

Os dados apurados foram expostos em tabelas, gráficos e demonstrados em discursos. Para evidenciar melhor a experiência de aprendizado foram feitas comparaçōes entre as informaçōes fornecidas antes e depois do processo. Os integrantes são identificados pela letra $I$ acrescido de um numeral: $I_{1}, I_{2}, I_{3}, I_{4}$ e assim por diante.

\section{ANÁLISE DE DADOS}

GRÁFICO 1: Distribuição da amostra, segundo nivel de escolaridade e tempo no projeto. Departamento de Enfermagem/UFC. Fortaleza - CE. Abril de 1998.

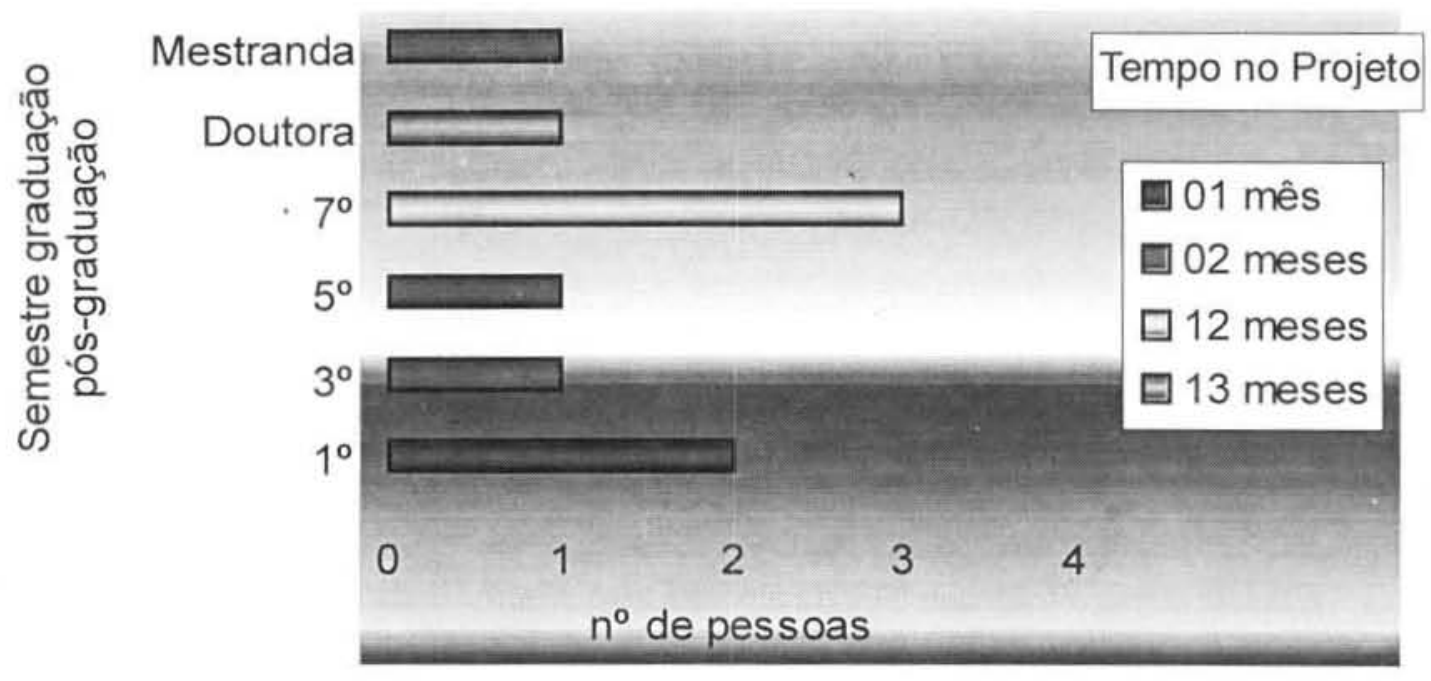

Diante dos dados, percebe-se que boa parte da amostra é composta por acadêmicos de enfermagem do $7^{\circ}$ semestre, correspondendo aproximadamente a $33,3 \%$ do total, estando estes também com maior tempo de atuação dentro do projeto (12 meses). Além disso, é notável que o projeto é composto por alunos de diversos niveis, inclusive da pós-graduação e que oito $(87,7 \%)$ da amostra estão inseridos no projeto há um ano ou menos, o que é considerado pouco tempo, embora, segundo Campos (1987) a maneira de aprender e o ritmo do processo seja variável de individuo para individuo, frente ao aspecto pessoal da aprendizagem. Isso significa que as caracteristicas intelectuais do grupo em questão poderão trazer um ritmo diferenciado na execuçăo do desenvolvimento educativo, considerando a experiência anterior de cada membro. 
TABELA 1: Definição de PA no $1^{\circ}$ e $2^{\circ}$ momentos do processo de ensino-aprendizagem. Departamento de Enfermagem/UFC. Fortaleza - CE. Abril de 1998.

\begin{tabular}{|c|c|c|}
\hline$\underbrace{\text { Período }}_{\text {Def. de PA }}$ & $1^{\circ}$ & $2^{\circ}$ \\
\hline Correta & 08 & 09 \\
\hline Parcialmente correta & 01 & - \\
\hline Total & 09 & 09 \\
\hline
\end{tabular}

A tabela descreve a correção ou não da definição de $\mathrm{PA}$ no $1^{\circ} \mathrm{e} 2^{\circ}$ momentos do processo de ensino-aprendizagem. A orientação da aprendizagem tem como meta primordial alterar o comportamento do educando. Tal propósito leva, necessariamente, a uma tomada de decisão sobre a natureza da mudança que se pretende realizar (Turra et al., 1975). Seguindo a linha de raciocínio dos autores e com os dados supracitados podemos inferir que houve mudança de comportamento, visto que, um dos participantes definiu PA no $1^{\circ}$ momento de forma parcialmente correta e no $2^{\circ}$ momento o fez de forma correta.

TABELA 2: Distribuição da amostra, segundo verificação da PA. Departamento de Enfermagem/UFC. Fortaleza - CE. Abril de 1998.

\begin{tabular}{lcc} 
Verificação da PA & $N^{\circ}$ & $\%$ \\
Sim, raras vezes & 02 & 22 \\
Sim, freqüentemente & 06 & 67 \\
Não, nunca & 01 & 11 \\
\hline Total & 09 & 100 \\
\hline
\end{tabular}

A tabela acima retrata que $89 \%$ da amostra teve alguma experiência prévia na técnica de verificação da PA. Sendo que destes, $67 \%$ verificavam freqüentemente e $22 \%$ esporadicamente. Situação contribuidora para o processo ensino-aprendizagem da técnica correta de verificação da PA.

Campos (1987) refere que cada nova aprendizagem soma novos elementos à experiência prévia, num contexto gradativo e ascendente. Com essa reflexão, queremos enfatizar que a vivência do grupo no que tange a aferição da pressão arterial foi facilitadora do processo de ensino-aprendizagem desenvolvido. Dentro deste mesmo contexto, Du Gas (1988) retrata que a aprendizagem é possibilitada quando o aprendiz entende as definições fundamentais em que é baseado o tema a ser aprendido. 
QUADRO 1: Fatores de erro na verificação da PA citados pelos respondentes antes e após a reciclagem. Departamento de Enfermagem/UFC. Fortaleza - CE. Abril de 1998.

\begin{tabular}{|c|c|}
\hline Inquietude/tensão $\left(I_{1}\right)$ & $\begin{array}{l}\text { Estado do paciente, a maneira como ele está } \\
\text { vestido, posição da pessoa }\left(\mathrm{I}_{1}\right)\end{array}$ \\
\hline Agitação $\left(\mathrm{I}_{2}\right)$ & Fumo, álcool, sal, lipídeos $\left(\mathrm{I}_{2}\right)$ \\
\hline $\begin{array}{l}\text { Álcool, fumo, café, ambiente, estresse, } \\
\text { exercícios físicos, bexiga cheia, tamanho do } \\
\text { manguito }\left(\mathrm{I}_{3}\right)\end{array}$ & $\begin{array}{l}\text { Tamanho do manguito, altura do braço, } \\
\text { ansiedade, fumo, café, hora, ambiente, } \\
\text { estresse, exercícios físicos, bexiga cheia }\left(\mathrm{I}_{3}\right)\end{array}$ \\
\hline $\begin{array}{l}\text { Ansiedade, hora, posição, roupas apertadas, } \\
\text { alimentação }\left(\mathrm{I}_{4}\right)\end{array}$ & $\begin{array}{l}\text { Ambiente, posição, horário, ansiedade, } \\
\text { roupas apertadas }\left(\mathrm{I}_{4}\right)\end{array}$ \\
\hline $\begin{array}{l}\text { Alimentação, gravidez, posição, exercícios } \\
\text { físicos, estresse e obesidade }\left(I_{5}\right)\end{array}$ & $\begin{array}{l}\text { Fumo, alimentação, posição, exercícios } \\
\text { físicos, gravidez, estresse, obesidade, álcool e } \\
\text { outras drogas }\left(\mathrm{I}_{5}\right)\end{array}$ \\
\hline Emoção, estresse, tensão e obesidade $\left(\mathrm{I}_{6}\right)$ & Material utilizado, emoção, estresse $\left(\mathrm{I}_{6}\right)$ \\
\hline $\begin{array}{l}\text { Fatores relacionados ao examinador, ao } \\
\text { cliente e aparelhos }\left(\mathrm{I}_{7}\right)\end{array}$ & $\begin{array}{l}\text { Fatores relacionados ao examinador, ao } \\
\text { cliente e aparelhos }\left(\mathrm{I}_{7}\right)\end{array}$ \\
\hline $\begin{array}{l}\text { Estresse, barulho, agitação, roupas apertadas } \\
\text { e pessoas conversadeiras }\left(\mathrm{I}_{8}\right)\end{array}$ & $\begin{array}{l}\text { Estresse, barulho, lugares agitados, roupas } \\
\text { apertadas e pessoas que gostam de conversar } \\
\left(\mathrm{I}_{8}\right)\end{array}$ \\
\hline $\begin{array}{l}\text { Alimentação, } \\
\text { emocional, est }\end{array}$ & $\begin{array}{l}\text { Alimentação, medicamentos, estado } \\
\text { emocional, estresse e fumo. }\left(I_{9}\right)\end{array}$ \\
\hline
\end{tabular}

O quadro acima demonstra fatores de erro na verificação da PA, citados pela clientela antes e após o processo de ensino-aprendizagem. Vale salientar que utilizamos a identificação referida anteriormente para categorização do grupo. É importante observar também que a numeração indica coincidência da clientela no primeiro e segundo momento.

A aferição da PA é um procedimento simples e fácil de ser realizado. Contudo, determinados principios devem ser atendidos no momento da técnica, evitando-se, assim, fatores de erro. Para a medida precisa da PAe, conseqüentemente, o diagnóstico correto da hipertensão , é imprescindivel adotar os cuidados e os critérios relacionados ao observador, ao paciente, ao ambiente e ao equipamento (Pierin; Santello; Mion Jr., 1997).

Diante desta realidade, observa-se que a clientela, de maneira geral, citou alguns elementos que interferem, ou melhor, que podem causar erros na técnica de verificação da PA. Percebe-se, contudo, que dentre os fatores de erro relacionados à medida da PA o mais referido foi relativo ao paciente. É importante que se considere também o observador, o ambiente e o equipamento.

Apesar de haver padronização na medida da PA e ser uma prática rotineira, ainda, ocorre erro na aferição, visto que, muitos profissionais a fazem de maneira incorreta.

Fazendo uma análise comparativa com o quadro do "antes", pode-se perceber, de maneira geral, uma complementariedade de conhecimentos. Havendo referência mais significativa ao ambiente, cliente e equipamento. Contudo, observa-se, ainda uma lacuna relativa ao observador, que foi citado por apenas uma pessoa, tanto antes quanto após o processo de ensinoaprendizagem. 
TABELA 3: Distribuição do grupo, segundo dificuldade na verificação da PA, considerando o momento pré e pós reciclagem. Departamento de Enfermagem/UFC. Fortaleza - CE. Abril de 1998.

\begin{tabular}{l|cc}
\multicolumn{1}{r|}{ Período } & Antes & Depois \\
\cline { 1 - 1 } Dificuldades & & \\
Sim & 04 & 05 \\
Não & 04 & 04 \\
Não respondeu & 01 & -- \\
\hline Total & 09 & 09 \\
\hline
\end{tabular}

Diante dos resultados acima, podemos demonstrar que antes da realização do processo quatro pessoas do grupo demonstraram dificuldades na aferição da PA e posteriormente cinco. Resultado esse que nos revela que a partir do processo ensino-aprendizagem é possivel ocorrer dúvidas e questionamentos. Contribuindo, assim, para o aprimoramento do processo, com envolvimento bilateral de educando e educador.

Com base nestas considerações observa-se que no momento do aprendizado ocorreu uma busca ascendente de conhecimento relativo às dificuldades encontradas na aferição da pressão arterial. Considerando Du Gas (1988), a aprendizagem provoca mudança nos processos de pensamento, açōes ou atitudes do aprendiz.

TABELA 4: Dificuldades encontradas pelo grupo, antes e após a realização do processo de ensino-aprendizagem. Departamento de Enfermagem/UFC. Fortaleza -CE. Abril de 1998.

\begin{tabular}{lcc} 
Dificuldades & Antes & Após \\
& $\mathrm{n}$ & $\mathrm{n}$ \\
\hline Não consegue ouvir a diastólica & 01 & - \\
$\begin{array}{l}\text { Diferença entre o ensinado e o utilizado na } \\
\text { prática }\end{array}$ & 01 & 01 \\
Conversas paralelas, pressa e cansaço & 01 & 01 \\
Aparelhos não calibrados & 01 & 01 \\
Achar o pulso radial/pulsação & - & 02 \\
\hline Total & 04 & 05 \\
\hline
\end{tabular}

A tabela revela as dificuldades encontradas pelo grupo no $1^{\circ}$ momento. As dificuldades relatadas pela clientela mostraram mais uma vez que os fatores relacionados ao ambiente, ao observador e ao equipamento, são aqueles que podem causar erros na aferição da PA, e consequentemente dificultam o processo de mensuração.

É válido ressaltar que no $2^{\circ}$ momento, tivemos a manutenção de três dificuldades, correspondendo a "diferença entre o ensinado e o aprendido", "conversas paralelas, pressa e cansaço" e "aparelhos não calibrados". Além destas surgiram mais duas: "achar o pulso radial" e "achar pulsação". 
Observa-se com este resultado que o processo de aprendizagem é algo muito complexo e sua avaliação algo bastante complicado.

TABELA 5: Contribuiçăo do processo utilizado para o aprimoramento da técnica de verificação da PA. Departamento de Enfermagem/UFC. Fortaleza - CE. Abril de 1998.

\begin{tabular}{lc} 
Contribuição & $\mathrm{N}^{\circ}$ \\
Sim & 09 \\
Não &.-- \\
\hline Total & 09 \\
\hline
\end{tabular}

Podemos referir que o processo educativo aplicado foi importante para a clientela envolvida, visto que a totalidade dos participantes avaliou a reciclagem como válida para o aprimoramento adequado do conhecimento relativo à técnica de verificação da PA.

A avaliação é a maneira de pesquisar a distância percorrida pelo aprendiz, levando em consideração objetivos preestabelecidos. Se os objetivos foram enuciados especificamente em termos do que se espera que o educando seja capaz de realizar ao término do ensino, a avaliação de sua aprendizagem torna-se relativamente simples (Du Gas, 1988).

\title{
CONSIDERAÇÖES FINAIS
}

Diante do exposto, conclui-se que:

- A técnica de verificação da PA no grupo de pesquisa em estudo foi aprimorada;

-O conhecimento apreendido sobre verificação da PA pela clientela mostrou-se mais incompleto e difuso no momento anterior ao processo de ensino aprendizagem.

-Considerando o momento posterior ao processo desenvolvido, pode-se dizer que houve um aperfeiçoamento do conhecimento da técnica de verificaçăo da PA, mas é válido ressaltar que tal capacitação não pode ser entendida como totalizadora, ou seja, com o dominio completo da técnica, pela própria caracteristica inacabada do processo ensino-aprendizagem e postura reflexiva do individuo.

\begin{abstract}
The nurse is considered the professional that obtains more trustworthy data in the measures insinuations of arterial pressure for inducing less reactions of alert in the customer. generating of values falsely high. Even so, a lot of times, she presents spaces in the knowledge of the subject, so much in the technical aspects, as in the anátomo-physiologic ones. That prioritizes the development of teaching strategies, seeking to minimize flaws and to turn the effective learning process. Objectifying to promote the knowledge of the technique of measurement of the arterial pressure close to a research group, it took place a reciclation and avaliation questionnaire of the knowledge of the group was applied before and after the same. The effectiveness of that method was evidenced, because $100 \%$ of the sample (nine) they referred contribution for better of the fundamentation theoretical-practice, decreasing difficulties originating from of the verification of the arterial pressure. It is ended that, being the education actions in extremely valid and necessary health, the male nurse, as educator that is, needs to recycle its knowledge and its better techniques continually.
\end{abstract}

KEYWORDS: teaching-learning, arterial pressure, nursing. 
RESUMEN: La enfermera es considerada la profesional que obtiene datos más fidedignos en las medidas indirectas de presión arterial (P.A.) por inducir menos reacciones de alerta en el cliente, generadoras de valores falsamente elevados. Sin embargo, muchas veces, ella presenta lagunas en el conocimiento del asunto, tanto en los aspectos técnicos, como en los anatómicos y físiológicos. Esto torna prioritario el desarrollo de estrategias de enseñanza, con miras a disminuir los errores y volver efectivo el proceso de aprendizaje. Objetivando promover el conocimiento de la técnica de medida de la presión arterial (P.A.) junto a un grupo de pesquisa, se realizó un reciclaje y se aplicó un cuestionario de evaluación del grupo antes y después del mismo. Se puso en evidencia la eficacia del método, pues $100 \%$ de la muestra (nueve) refirió contribución para la mejoria de los fundamentos teóricos y prácticos, disminuyendo las dificultades originadas de la verificación de la presión arterial. Se concluye que, siendo las acciones de educación en salud de extrema necesidad y validez, el enfermero, como educador que es, necesita reciclar su conocimiento y mejorar sus técnicas continuamente.

PALAVRAS LLAVE: enseñanza - aprendizaje, presión arterial, enfermeria.

\section{REFERÊNCIAS BIBLIOGRÁFICAS}

ARAUJO, T. L. de. Medida indireta da pressão arterial: caracterização do conhecimento_do enfermeiro. Sāo Paulo, 1994. Tese (Doutorado). Escola de Enfermagem. Universidade de São Paulo.

ARAUJO, T. L. de., ARCURI, E.A.M., MARTINS, E. Instrumentaçào na medida da pressāo arterial: aspectos históricos, conceituais e fontes de erro. Rev. Esc. Enf._USP, v. 32, n. 1 , p. 33-41, abr. 1998.

ARCURI,E.A.M. Medida indireta da pressão: revisão. Rev.ESc.Enf.USP., v.23, p.163-74, 1989.

BORDENAVE, J.D;; PEREIRA, A. H. Estratégias de ensino-aprendizagem. Petropólis: Vozes, 1977. p. 15-57.

CAMPOS, D. M. de S. Psicologia da aprendizagem. 23. ed. Petrópolis: Vozes, p. 28-38, 1987.

COLLET, N., ROCHA, S. M. M. Transformações no ensino das técnicas em_enfermagem pediátrica. Goiânia: AB, 1996. 146 p.

DU GAS, B. W. Enfermagem prática. 4. ed. Rio de Janeiro: Guanabara, 1988. p.115-136.

GAGNE, R. M. Como se realiza a aprendizagem. Rio de Janeiro: Livro Técnico, 1973. p. 1-62

HAS. Hipertensão arterial sistêmica. Ciclo de atualização cientifica, série 3. São Paulo: Boehringer de Angeli, 1996

HILL, M.; McCOMBS, N.J. O desafio do controle da hipertensão arterial. Simpósio sobre hipertensăo Cl. Enf. Am. Norte, Jun 198. Hipertensāo. Rio de Janeiro: Interamericana. p. 281-291 e 351-365

LOLIO, C.A. Epidemiologia da hipertensāo arterial. Rev. Saúde Públ. Săo Paulo, v24, p.425-32, 1990.

MANCIA, G. et al. Alerting reaction and rise in blood pressure during measurement by physician and nurse. Hypertension, v.9, p.200-15, 1987.

MIZUKAMI, M. das G. N. Ensino: as abordagens do processo. Sāo Paulo: E.P.U., 1986. p. 85 - 103

NOVAES, M. H. Psicologia da educação e prática profissional. Petropólis (RJ): Vozes, 1992. p. 93 $-107$

PERLOFF, D. et al. Human blod pressure determination by sphygmomanometry. Circulation, v.88, n.5, p.2460-2470, parte I, nov. 1993.

PIERIN, A. M. G.; SANTELLO, J. L.; MION JÜNIOR, D. Medida da pressão arterial na clinica. In: AMODEO, C., LIMA, E. G., VAZQUEZ, E. C. Hipertensāo arterial. São Paulo: Sarvier, 1997. cap. 8, p. $73-82$

PORTO, C. C., RASSI, S. Sistema cardiovascular. In: PORTO, C. C. Semiologia_médica. RJ: Guanabara Koogan, 1990. Parte 6, seção 1, p.293-382.

TURRA, C.M.G. et al. Planejamento de ensino e avaliação. 4. ed. Porto Alegre: PUC, Emma. 1975. p. 123-155.

ZANELLA, M.T. et. al. Hipertensăo arterial essencial: conceito e etiopatogenia. RSCESP (Sāo Paulo), v. 2, n. 4, p. 18-22, jullago. 1992. 